\title{
Perfil de recém-nascidos pré-termo internados na unidade de terapia intensiva de hospital de alta complexidade
}

\author{
Profile of preterm newborn hospitalized in intensive care unit of high \\ complexity hospital
}

Caroline de Sousa Oliveira ${ }^{1}$, Gabriela Ay Casagrande ${ }^{1,2}$, Luanda Collange Grecco ${ }^{1}$, Marina Ortega Golin ${ }^{1}$

${ }^{1}$ Curso de Fisioterapia da Faculdade de Medicina do ABC (FMABC) - Santo André (SP), Brasil.

${ }^{2}$ Unidade de Terapia Intensiva Neonatal do Hospital Estadual Mário Covas (HEMC) - Santo André (SP), Brasil.

DOI: http://dx.doi.org/10.7322/abcshs.v40i1.700

\begin{abstract}
RESUMO
Introdução: A pesquisa epidemiológica consiste em alicerce para avanços nos cuidados da saúde. Nacionalmente, os estudos sobre caracterização de diferentes populações não são frequentes, principalmente no que concerne aos recém-nascidos pré-termo (RNPT). Objetivo: Caracterizar RNPT atendidos na Unidade de Terapia Intensiva (UTI) Neonatal do Hospital Estadual Mário Covas de Santo André (SP) pelo delineamento gestacional, perinatal e período de internação. Métodos: Trata-se de estudo transversal descritivo. Foram analisados 218 prontuários de todos os pacientes atendidos pelo serviço de fisioterapia no período de 2008 a 2011 e, então, delimitada a amostra consecutiva com 81 deles, que seguiram os critérios de inclusão e exclusão. Os dados foram organizados e tabulados pelo programa SPSS v.17.0. O teste do $\chi^{2}$ foi utilizado para comparar as frequências de complicações neonatais. Resultados: As genitoras tinham idade média de 25,7 anos; destas, 68,6\% realizaram pré-natal. As intercorrências gestacionais foram observadas em 37,3\% (as infecções do trato urinário foram as mais comuns, em 28\%). A média do peso ao nascimento foi $1.478,6 \mathrm{~g}$, e a de idade gestacional, de 30,8 semanas. O tempo médio de internação dos RNPT foi de 36 dias. As patologias respiratórias ocorreram em $93,8 \%$, sendo o desconforto respiratório precoce a mais comum $(95,2 \%)$. RNPT que necessitaram de ventilação mecânica foram 97,6\% e as patologias neurológicas foram menos frequentes $(9,87 \%)$. Conclusão: As complicações respiratórias foram frequentes nessa população e associadas significantemente com idade gestacional inferior a 32 semanas. Longos períodos de internação após o nascimento estão intimamente relacionados com baixo peso e idade gestacional ao nascimento.
\end{abstract}

Palavras-chave: trabalho de parto prematuro; prematuro; doenças do prematuro.

\begin{abstract}
Introduction: Epidemiological research consists offoundationfor advances in health care. Nationally, studies on characterization of different populations are not common, especially in relation to newborn preterm infants (PTI). Objective: Characterize the newborn preterm infants attended in the Neonatal Intensive Care Unit of the Hospital Estadual Mário Covas in Santo André the delineation of the breast profile, gestational, perinatal and period of hospitalization. Methods: This is a descriptive cross-sectional study. We analyzed 218 medical records of all patients seen by the physiotherapy service from 2008 to 2011 and then bounded to the consecutive sample of 81 of them, which followed the inclusion and exclusion criteria. The data were organized and tabulated with SPSS v.17.0 program. The chi-square test was used to compare the frequency of neonatal complications. Results: The mothers had a mean age of 25.7 years, and $68.6 \%$ received prenatal care. The pregnancy complications were observed in $37.3 \%$, with the most common urinary tract infections in $28 \%$. The mean birth weight was $1478.6 \mathrm{~g}$ and gestational age of 30.8 weeks. The average length of stay of preterm infants was 36 days. Respiratory disorders occurred in $95.2 \%$, with early respiratory distress the most common (95.2\%). PN requiring mechanical ventilation were $97.6 \%$ and neurological disorders were less frequent (9.87\%). Conclusion: Respiratory complications were common in this population and significantly associated with gestational age less than 32 weeks. Prolonged hospitalization after birth is closely related to low birth weight and gestational age at birth.
\end{abstract}

Keywords: obstetric labor, premature; infant, premature; infant, premature, diseases. 


\section{INTRODUÇÃO}

As expectativas familiares quanto a uma gestação tranquila e a chegada de um bebê saudável muitas vezes não se concretizam perante a necessidade de parto prematuro. A chegada de um recém-nascido pré-termo (RNPT), como é denominado aquele que nasce com idade gestacional igual ou inferior a 37 semanas $^{1}$, geralmente é associada a aflições oriundas das complicações que culminaram na antecipação do parto ${ }^{2}$.

A prematuridade está diretamente relacionada a causas gestacionais maternas e condições socioeconômicas desfavoráveis, uma vez que a falta de informação sobre os cuidados adequados durante a gestação, as condições precárias de assistência à saúde e o acompanhamento pré-natal insatisfatório são potencialmente prejudiciais ao desenvolvimento embrionário ${ }^{2,3}$.

Nesse contexto, torna-se necessário o emprego de cuidados especializados durante e após o parto, já que esses RNPT são muito vulneráveis a manipulações e ao desenvolvimento de patologias ${ }^{2,4,5}$. Esse suporte hospitalar é de extrema importância, porém a permanência no hospital deve ser reduzida ao máximo visando minimizar os riscos de mortalidade e morbidade . $^{\circ}$

Os distúrbios respiratórios correspondem às intercorrências mais comuns nesse período, resultantes da imaturidade do sistema respiratório e da incapacidade de produção de surfactante ${ }^{7,8}$. As complicações da prematuridade também podem fragilizar o sistema nervoso central (SNC) e gerar afecções cerebrais. Dentre estas, as mais comuns são as hemorragias peri e intraventriculares e a leucomalácia periventricular ${ }^{9-11}$.

Em decorrência da exposição a diversos fatores de risco para lesões neurológicas, é esperado que os RNPT apresentem atraso no desenvolvimento neuropsicomotor, comprometimento das habilidades motoras, cognitivas, perceptuais, de linguagem ${ }^{3,4,12}$, entre outros, sendo que muitos desses prematuros nascem com baixo peso, que consiste em preditor de complicações no crescimento e desenvolvimento infantil ${ }^{10,12,13}$.

Estudos evidenciam que perante esse panorama se faz necessária a intervenção precoce para minimizar as consequências deletérias no desenvolvimento dessas crianças ${ }^{6}$. Contudo, a estratégia mais efetiva na tentativa de garantir um desenvolvimento saudável seria a prevenção tanto da prematuridade quanto de suas complicações.

Nesse aspecto, a pesquisa epidemiológica assume notável importância, pois consiste em alicerce para avanços nos cuidados da saúde humana, principalmente daqueles que têm a vida toda pela frente. Dessa forma, estudos que estabeleçam o perfil de RNPT em diferentes ambientes e contextos são relevantes, pois auxiliam na compreensão de suas necessidades especiais e particulares, contribuindo para que os cuidados no período neonatal sejam diferenciados e específicos e que a prevenção possa tornar-se uma realidade.

O presente estudo teve como objetivo caracterizar RNPT atendidos na Unidade de Terapia Intensiva (UTI) Neonatal de hospital de alta complexidade pelo delineamento do perfil materno, gestacional, perinatal e período de internação.

\section{MÉTODOS}

Trata-se de estudo transversal descritivo. Foram consultados 218 prontuários de todos os RNPT atendidos pelo Serviço de Fisioterapia da UTI Neonatal do Hospital Estadual Mário Covas de Santo André (HEMC) (SP) no período de 2008 a 2011. Após triagem inicial dos prontuários, 81 se enquadraram nos critérios de inclusão e foram analisados neste estudo.

Para inclusão, foram adotados os seguintes critérios: idade gestacional ao nascimento igual ou inferior a 37 semanas e internação na UTI Neonatal no período que compreendeu a coleta de dados. Os de exclusão foram prontuários com dados insuficientes para contemplar o protocolo da pesquisa e presença de síndromes e más-formações congênitas.

A coleta de dados foi iniciada após aprovação do projeto pelo Comitê de Ética em Pesquisa da Faculdade de Medicina do ABC (FMABC), sob o protocolo no 195.458/2013.

O primeiro passo foi o levantamento dos RNPT, via consulta da estatística dos atendimentos do Serviço de Fisioterapia da UTI Neonatal. Após delimitação da amostra, a consulta direta aos prontuários foi iniciada.

O protocolo para coleta de dados foi elaborado para a presente pesquisa com as seguintes informações: idade materna, realização de pré-natal e número de consultas, intercorrências no período gestacional, tipo de parto, apgar do $5^{\circ}$ minuto, uso de surfactante, idade gestacional e peso ao nascimento, resultados de ultrassom transfontanela, presença de patologias neurológicas e respiratórias no período neonatal, uso e permanência em ventilação mecânica, uso e permanência em oxigenoterapia, presença de processos infecciosos e tempo de internação.

Para análise das variáveis categóricas, foi utilizada tabela de frequência (relativa e absoluta). A estatística descritiva (média/desvio padrão) foi adotada para as variáveis quantitativas contínuas. Os dados foram organizados e tabulados pelo programa SPSS v.17.0. O teste do $\chi^{2}$ foi utilizado para comparar as frequências do desenvolvimento de doenças neurológicas, doenças respiratórias, sepse e internação prolongada de acordo com o peso ao nascimento e idade gestacional. Os valores de $\mathrm{p}<0.05$ foram considerados significantes.

\section{RESULTADOS}

As genitoras tinham idade média de 25,7 anos (destas, 69,1\% realizaram pré-natal).

As intercorrências gestacionais ocorreram em $67,9 \%$ dos casos e as infecções do trato urinário (ITU) foram as mais comuns, em $28 \%$ das gestantes, seguidas pela doença hipertensiva específica da gravidez (DHEG), em 17\%. Dessas, 9\% apresentaram hipertensão arterial sistêmica (HAS), 4\%, diabetes mellitus, 2\%, oligoamnio e síndrome HELLP. Entre as menos frequentes estavam obesidade, depressão, retardo do crescimento intrauterino, polidramnio, pneumonia, descolamento prematuro de placenta, 
entre outros, que ocuparam $1 \%$. O uso de drogas ilícitas foi observado em $3 \%$ dos casos.

As principais características dos RNPT estão descritas na Tabela 1.

A média do peso ao nascimento foi $1.478,6 \mathrm{~g}$, e a da idade gestacional, de 30,8 semanas. O uso de surfactante foi necessário em 53\%. No período neonatal, o tempo médio de internação dos RNPT foi de 36 dias. As patologias respiratórias ocorreram em 93,8\% dos RNPT, sendo a síndrome do desconforto respiratório

Tabela 1: Características dos recém-nascidos pré-termo, maternas, gestacionais e do período neonatal (Hospital Estadual Mário Covas, 2008 a 2011)

\begin{tabular}{|l|c|c|}
\hline Características & $\mathbf{n}$ & $\%$ \\
\hline $\begin{array}{l}\text { Sexo } \\
\text { Masculino }\end{array}$ & 42 & 51,8 \\
\hline Feminino & 39 & 48,1 \\
\hline Caracterização clínica & Sim & Não \\
\hline Patologias respiratórias & $\mathbf{n}(\%)$ & $\mathbf{n}(\%)$ \\
\hline Ventilação mecânica & $76(93,8)$ & $5(6,1)$ \\
\hline Oxigenoterapia & $73(90,1)$ & $8(9,8)$ \\
\hline Sepse & $59(72,83)$ & $22(27,1)$ \\
\hline Patologias neurológicas & $20(24,6)$ & $61(75,8)$ \\
\hline Outras características & $8(9,87)$ & $73(90,1)$ \\
\hline Idade materna (anos) & Média & Desvio padrão \\
\hline Número de consultas pré-natais & 25,7 & 7,6 \\
\hline Idade gestacional (semanas) & 4,6 & 4,1 \\
\hline Peso ao nascer (gramas) & 30,8 & 3,1 \\
\hline Apgar 5o minuto & 1478,6 & 558,2 \\
\hline Dias na ventilação mecânica & 7 & 2,1 \\
\hline Internação (dias) & 17,7 & 16,8 \\
\hline & 36 & 32,2 \\
\hline
\end{tabular}

(SDR) a mais comum $(95,2 \%)$, seguida por pneumonia $(34,5 \%)$, displasia broncopulmonar (9,8\%), apneia (4\%), insuficiência respiratória e hemorragia pulmonar (3\%), pneumonia, pneumotórax e atelectasia (1\%). Os RNPT que necessitaram de ventilação mecânica foram 90,1\%. Já as patologias neurológicas foram menos frequentes $(9,87 \%)$, sendo que hemorragias peri e intraventriculares estavam presentes em 3,6\%, e traumatismo crânio encefálico, em $1 \%$. Contudo, $85,1 \%$ dos prontuários não apresentavam registros de resultados de ultrassom transfontanela.

O peso ao nascimento e a idade gestacional são considerados fatores de risco determinantes da evolução do recém-nascido. Dessa forma, optou-se por verificar a associação de tais fatores com o desenvolvimento de doenças neurológicas e respiratórias, sepse e tempo de internação, conforme mostrado nas Tabelas 2 e 3 .

\section{DISCUSSÃO}

O nascimento prematuro é um notável problema de saúde perinatal em todo o mundo e a maior causa de mortalidade infantil no Brasil, sendo que as condições perinatais, na maioria das vezes, associam-se à prematuridade ${ }^{13-15}$.

Um estudo analisou 1.006 mulheres que deram à luz a recém-nascidos vivos e verificou que o trabalho de parto prematuro ocorreu em $40 \%$ dos casos, fatos que evidenciam sua alta prevalência e a necessidade de atenção especial no que se refere ao período gestacional ${ }^{15,16}$. Não menos impactantes são suas consequências, pois o RNPT pode sofrer complicações, visto que a prematuridade aumenta o risco de dificuldades de adaptação à vida extrauterina, principalmente devido à imaturidade anatômica e fisiológica ${ }^{1}$.

Tabela 2: Associação do peso ao nascimento com: doenças respiratórias e neurológicas, sepse e tempo de internação (Hospital Estadual Mário Covas, 2008 a 2011)

\begin{tabular}{|c|c|c|c|c|c|}
\hline \multirow{3}{*}{ Fatores analisados } & \multicolumn{4}{|c|}{ Peso ao nascimento } & \multirow{3}{*}{ Valor $\mathbf{p}^{*}$} \\
\hline & \multicolumn{2}{|c|}{ Inferior a $1.500 \mathrm{~g}$} & \multicolumn{2}{|c|}{ Superior a $1.500 \mathrm{~g}$} & \\
\hline & $\underset{n(\%)}{\operatorname{Sim}}$ & $\begin{array}{c}\text { Não } \\
\text { n (\%) }\end{array}$ & $\begin{array}{l}\text { Sim } \\
\text { n (\%) }\end{array}$ & $\begin{array}{l}\text { Não } \\
\text { n (\%) }\end{array}$ & \\
\hline Sepse & $10(12,34)$ & $28(34,56)$ & $10(12,34)$ & $33(40,74)$ & 0,634 \\
\hline Doenças neurológicas & $4(4,93)$ & $34(41,97)$ & $4(4,93)$ & $39(48,14)$ & 0,608 \\
\hline Doenças respiratórias & $35(43,20)$ & $3(3,70)$ & $41(50,61)$ & $2(2,46)$ & 0,507 \\
\hline Tempo de internação >30 dias & $21(25,92)$ & $17(20,98)$ & $13(16,04)$ & $30(37,03)$ & 0,002 \\
\hline
\end{tabular}

${ }^{*} \mathrm{p}<0,05$; teste do $\chi^{2}$

Tabela 3: Associação da idade gestacional ao nascimento com: doenças respiratórias e neurológicas, sepse e tempo de internação(Hospital Estadual Mário Covas, 2008 a 2011)

\begin{tabular}{|c|c|c|c|c|c|}
\hline \multirow{3}{*}{ Fatores analisados } & \multicolumn{4}{|c|}{ Idade gestacional ao nascimento } & \multirow{3}{*}{ Valor $\mathrm{p}^{*}$} \\
\hline & \multicolumn{2}{|c|}{ Inferior a 32 semanas } & \multicolumn{2}{|c|}{ Superior a 32 semanas } & \\
\hline & $\begin{array}{c}\text { Sim } \\
n(\%)\end{array}$ & $\begin{array}{c}\text { Não } \\
\text { n (\%) }\end{array}$ & $\underset{\text { n (\%) }}{\operatorname{Sim}}$ & $\begin{array}{l}\text { Não } \\
\text { n (\%) }\end{array}$ & \\
\hline Sepse & $15(18,51)$ & $29(35,80)$ & $5(6,17)$ & $32(39,50)$ & 0,553 \\
\hline Doenças neurológicas & $5(6,17)$ & $39(48,14)$ & $3(3,70)$ & $34(41,97)$ & 0,494 \\
\hline Tempo de internação $>30$ dias & $25(30,86)$ & $19(23,45)$ & $11(13,58)$ & $26(32,09)$ & 0,040 \\
\hline Doenças respiratórias & $41(50,61)$ & $3(3,70)$ & $35(43,20)$ & $2(2,46)$ & 0,002 \\
\hline
\end{tabular}

${ }^{*} \mathrm{p}<0,05$; teste do $\chi^{2}$ 
O acompanhamento pré-natal é essencial para monitorar o desenvolvimento intrauterino e a saúde materna. Sendo assim, o número de consultas presentes no período deveria ser diretamente relacionado à idade gestacional, mostrando que tal período foi acompanhado e se a gestação foi interrompida por alguma complicação. Aqui, das 81 gestantes analisadas, 69,1\% realizaram pré-natal, sendo o número médio de consultas de 4,6. Assim, é possível relacionar que o acompanhamento pré-natal pode ser decisivo para o parto prematuro. Entretanto, a média da idade materna foi de 25,7 anos, fator que pode ter contribuído para um pobre acompanhamento pré-natal.

As intercorrências gestacionais presentes em 67,9\% dos casos tiveram como destaque a infecção do trato urinário (ITU), que representa relevante fonte de complicações maternas. Sem contar que a bacteriúria assintomática acomete entre 2 e $10 \%$ de todas as gestantes $^{16}$. No estudo de Duarte, $29,3 \%$ das gestantes analisadas também apresentaram ITU, consistindo na intercorrência mais comum e com proporções de parto prematuro em $33 \%$ delas ${ }^{17}$.

De acordo a literatura, a ITU é um grande fator de risco para a prematuridade, podendo gerar complicações maternas, como obstrução urinária, corioamniorrexe prematura, anemia, corioamnionite, endometrite, pré-eclâmpsia e choque séptico, assim como perinatais, sendo prematuridade, infecção, leucomalácia periventricular, falência de múltiplos órgãos e óbito ${ }^{17}$.

Neste estudo a idade gestacional média ao nascimento foi de 30,8 semanas. Um aspecto decisivo deve ser apontado, pois é após esse período que se dá a formação de surfactante pelos pneumócitos tipo II, responsável por impedir o colabamento dos alvéolos quando em contato com o ar. Dos RNPT analisados, 53\% necessitaram de surfactante, o que pode estar relacionado com a idade gestacional, pois no momento em que iriam produzir surfactante a gestação foi interrompida, fato que também pode ter contribuído para o desenvolvimento de complicações respiratórias em 93,8\% dos casos.

De acordo com a literatura, a baixa frequência do uso de corticoesteroides no pré-parto e imediatamente após associa-se à maior incidência de SDR, pois pode evitar ou atenuar a sua gravidade $^{18}$. Embora a informação sobre o uso de corticoesteroide antenatal não tenha sido analisada aqui, mesmo 53\% dos RNPT tendo feito uso de surfactante, 95,06\% apresentaram SDR. Dados semelhantes foram encontrados no estudo realizado por Almeida, no qual 68\% dos RNPT precisaram de surfactante e 77\% também apresentaram SDR ${ }^{18}$.

A SDR continua sendo uma das complicações mais frequentes entre os neonatos com peso igual ou inferior a $1.500 \mathrm{~g}^{19}$, fato que se tornou evidente nessa população e pode estar associado ao elevado índice de necessidade de ventilação mecânica em $90,1 \%{ }^{20}$.

O registro de patologias neurológicas não foi tão comum na população estudada quanto o de respiratórias, presentes em $9,87 \%$, porcentagem inferior aos apontamentos de diversos trabalhos na literatura ${ }^{19}$, como os $21,2 \%$ dos RNPT analisados por Rade ${ }^{21}$. Todavia, nesse aspecto pode haver um viés, uma vez que em $85,1 \%$ dos prontuários não havia registros a respeito do ultrassom transfontanela, fato que pode ter subdimensionado essa proporção.

A presença de complicações neurológicas está relacionada à prematuridade, sendo considerados importantes fatores de risco a idade gestacional inferior a 30 semanas e $\mathrm{SDR}^{5,9}$, evidenciados também na população estudada.

A imunidade dos RNPT é deficiente pela imaturidade dos sistemas. Estudos comprovam que o baixo peso e a prematuridade são fatores de risco de destaque para quadros de sepse e que os principais sítios de infecção encontram-se no trato respiratório ${ }^{22}$. No presente estudo, 24,6\% dos RNPT desenvolveram sepse, porcentagem considerada pouco elevada, porém esperada, quando se leva em consideração a idade gestacional média de 30 semanas e o baixo peso.

Os dados acima vão de encontro aos referidos por Herrmann ${ }^{22}$, que, entretanto, refere o fato de que as intercorrências neonatais não teriam papel de destaque na gênese de sepse nos RNPT. Contudo, é necessário também considerar que a sepse tardia pode ser decorrente das condições hospitalares e da qualidade do atendimento. Os RNPT se tornam mais propensos a riscos infecciosos quando a internação é prolongada. Os pacientes estudados permaneceram internados 36 dias, em média. Diante disso, grande parte dos quadros infecciosos possivelmente foi evitada.

Todavia, não se pode desmerecer a importância da qualidade do atendimento prestado na unidade, fato que certamente contribuiu para curtos períodos de internação.

Enfim, condições gestacionais desfavoráveis podem propiciar a prematuridade e a necessidade de tratamento em UTI. Entretanto, o atendimento de qualidade no período neonatal pode ser diferencial, contribuindo para diminuir os índices de complicações.

Importante ressaltar que tanto a intervenção no período gestacional quanto as condições dos atendimento pré-natais adequadas podem minimizar ou até mesmo retardar o parto prematuro, evitando o nascimento antecipado desses pré-termos tão suscetíveis a complicações. Ainda, os cuidados despendidos nos casos de internação em UTI podem ser determinantes de um desfecho positivo para a qualidade de vida de RNPT.

O presente estudo apresentou limitações que podem ter prejudicado a delimitação do real perfil. Um fator de destaque foi a redução do número da amostra devido à ausência de registros dos dados coletados nos prontuários. Outro aspecto foi a seleção dos participantes da estatística dos atendimentos fisioterapêuticos da UTI, uma vez que o hospital não possui maternidade. Não sendo possível também determinar a proporção de partos prematuros e daqueles que não necessitaram de internação no período neonatal.

Em conclusão, as complicações respiratórias foram frequentes nessa população e associadas significantemente com idade gestacional inferior a 32 semanas. Longos períodos de internação após o nascimento estão intimamente relacionados com baixo peso e idade gestacional ao nascimento. 


\section{REFERÊNCIAS}

1. Salge AKM, Vieira AVC, Aguiar AKA, Lobo SF, Xavier RM, Zatta LT, et al. Fatores maternos e neonatais associados à prematuridade. Rev Enferm. 2009;11(3):642-46

2. Ramos HAC, Cuman RKN. Fatores de risco para prematuridade: pesquisa documental. Rev Enferm. 2009;13(2):297-4

3. Rosa Neto F, Caon G, Bissani C, Silva CA, Silva MSE. Características neuropsicomotoras de crianças de alto risco neurológico atendidas em um programa de follow-up. Pediatr Mod. 2006;42(2):79-85.

4. Tronchin DMR, Tsunechiro MA. Prematuros de muito baixo peso: do nascimento ao primeiro ano de vida. Rev Gaúcha Enferm. 2007;28(1):79-88.

5. Marba STM, Caldas JPS, Vinagre LEF, Pessoto MA. Incidência de hemorragia peri-intraventricular em recémnascidos de muito baixo peso: análise de 15 anos. J Pediatr. 2011;87(6):505-11. http://dx.doi.org/10.1590/S0021-75572011000600008

6. Formiga CKMR, Pedrazzani ES, Tudella E. Desenvolvimento Motor de lactentes pré-termo participantes de um programa de intervenção fisioterapêutica precoce. Rev Bras Fisioter. 2004; 8(3):239-45.

7. Freddi NA, Proença Filho JO, Fiori HH. Terapia com surfactante pulmonar exógeno em pediatria. J Pediatr. 2003;79(Supp.2):S205-12 http://dx.doi.org/10.1590/S0021-75572003000800010

8. Brunherotti MAA, Vianna JRF, Silveira CST. Diminuição da ocorrência de pneumotórax em recém-nascidos com síndrome de desconforto respiratório através de estratégias de redução de parâmetros ventilatórios. J Pediatr. 2003;79(1):75-80.

9. Marinho RS, Cardoso LA, Idalgo GF, Jucá SSH. Hemorragia periventricular, intraventricular e mecanismos associados à lesão em recém-nascidos pré-termos. Acta Fisiatr. 2007;14(3):154-58.

10. Neves LAT, Chehuen Neto JA, Kneipp D, Fonseca LG, Rosado MP, Barreto MRP. Hemorragia intracraniana no recém-nascido pré-termo: casuística da UTI Neonatal do Hospital Albert Sabin. HU Rev. 2007;33(2):47-52.

11. Abreu LC, Souza AMB, Oliveira AG, Selestrin CC, Guerra MSB, Ferreira $C$, et al. Incidência de hemorragia peri-intraventricular em recém-nascidos pré-termo e a relação com o peso ao nascer. Rev Bras Crescimento Desenvol Hum. 2007;17(2):24-30
12. Silveira RC, Procianoy RS. Lesões isquêmicas cerebrais no recémnascido pré-termo de muito baixo peso. J Pediatr. 2005;81(1):S23-32.

13. Beck S, Wojdyla D, Say L, BetranAP, Merialdi M, Requejo JH, et al. The worldwide incidence of preterm birth: a systematic review of maternal mortality and morbidity. Bull World Health Organ. 2010;88(1):31-8. http://dx.doi.org/10.2471/BLT.08.062554

14. Silveira MF, Santos IS, Barros AJD, Matijasevich A, Barros FC, Victora CG. Aumento da prematuridade no Brasil: revisão de estudos de base populacional. Rev Saúde Pública. 2008;42(5):957-64.

http://dx.doi.org/10.1590/S0034-89102008000500023

15. McElrath TF, Hecht JL, Dammann O, Boggess K, Onderkong A Markenson G, et al. Pregnancy disorders that lead to delivery before the 28th week of gestation: an epidemiologic approach to classification. Am J Epidemiol. 2008;168(9):980-89. http://dx.doi.org/10.1093/aje/kwn202

16. Duarte G, Marcolin AC, Quintana SM, Cavalli RC. Infecção urinária na gravidez. Rev Bras Ginecol Obstetr. 2008;30(2):93-100. http://dx.doi.org/10.1590/S0100-72032008000200008

17. Duarte G, Marcolin AC, Gonçalves CV, Quintana SM, Berezowsk AT, Nogueira AA, et al. Infecção urinária na gravidez: análise dos métodos para diagnóstico e do tratamento. Rev Bras Ginecol Obstet. 2002;24(7):471-77. http://dx.doi.org/10.1590/S0100-72032002000700007

18. Almeida MFB,GuinsburgR, Martinez FE, Procianoy RS, Leone CR, Marba STM, et al. Fatores perinatais associados ao óbito precoce em prematuros nascidos nos centros da Rede Brasileira de Pesquisas Neonatais. Arch Pediatr Urug. 2010; 81(2):112-20.

19. Malveira SS, Moraes NA, Chermont AG, Costa DLF, Silva TF. Recém-nascidos de muito baixo peso em um hospital de referência. Rev Para Med. 2006;20(1):41-5.

20. Suguihara $C$, Lessa AC. Como minimizar a lesão pulmonar no prematuro extremo: propostas. J Pediatr. 2005;81(1):S69-78.

21. Rades E, Bittar RE, Zugaib M. Determinantes diretos do parto prematuro eletivo e os resultados neonatais. RBGO. 2004;26(8):655-62.

22. Herrmann DMML, Amaral LMB, Almeida SC. Fatores de risco para o desenvolvimento de sepse neonatal tardia em uma unidade de terapia intensiva. Pediatr. 2008;30(4):228-36. 